\title{
Explosion fragments by numbers
}

What happens when an explosive shell blows up, or when an array of galaxies is formed in a "big bang"? There will be fragments, but calculating their properties is a complicated matter.

EXPLODING shells kill people unfortunate enough to be nearby mostly by the impact of fragments of their casings, which is why shell-designers must arrange that there are as many lethal fragments as possible. Plainly, there are complicated trade-offs to make. To kill a person, a fragment must carry enough momentum to cause lethal damage, to a first approximation just that momentum needed to punch a hole in the human body. But momentum in excess of that threshold is a waste of kinetic energy.

So the optimum solution (if that is the phrase) is that in which there are as many fragments as possible with threshold momentum. The snag is that the total kinetic energy to send the fragments flying is the explosive power of the shell less whatever energy is spent in the fragmentation of the case: more fragments mean that less kinetic energy is available.

The military have been occupied with these recondite questions at least since early in the Second World War. So much is clear from the list of references for an article by Brad Lee Holian from the Los Alamos National Laboratory and Dennis E. Grady from the Sandia National Laboratories, whose earliest reference is to an unpublished British Ministry of Supply report in 1943 by N.F. Mott (now Sir Nevill Mott) and E.H. Linfoot (Phys. Rev. Lett. 60, 1355; 1988).

Mott explains that part of his wartime work in what was then called operations research required a relationship between lethality and fragment size, and that curiosity impelled him and Linfoot to look into the expected distribution of fragments with size. That is the aspect of this distant but not forgotten work to which Holian and Grady refer.

Luckily, there is more to be said about fragmentation than the lethality of exploding shells. Holian and Grady also refer to the big bang with which the Universe is supposed to have begun, and show that their calculations have something to say about the present distribution of galaxies with mass. But there are many other applications, while their technique is at once novel and potentially important. It may also encourage people by suggesting that there is still hope of an analytical treatment of the problem.

The essentials of the technique are borrowed from molecular dynamics and are applied, for the sake of making the problem tractable, to a two-dimensional structure in which elements of the material (think of them as atoms) are enclosed in a square piece of a square lattice. The particular calculation described rests on the assumption that elements of the fragmenting material interact with their nearest neighbours only by means of a LennardJones potential - the difference between a repulsive inverse sixth-power potential and an attractive inverse twelfth-power potential. By imposing periodic boundary conditions, the square piece of the square lattice can represent an infinite twodimensional sheet. To obtain worthwhile statistics, it is necessary to put as many as 4,200 elements in each expanding square.

To make the molecular dynamics work, Holian and Grady simply set the lattice expanding isotropically, so that the initial relative velocity of an arbitrary particle is a linear function of its distance from the point of reference, but the computer codes arrange that, if a particle leaves the basic lattice block by crossing one edge, another will enter with the same velocity at the corresponding point of the opposite edge. The particles originally have a certain amount of energy, or a non-zero temperature. Once the system has been set moving, the particles simply obey Newton's laws, which is to say that the fragmentation is adiabatic.

Qualitatively, the results are as neat as one could hope. The first stages of the expansion entail merely that the underlying chequerboard structure stretches as atoms are pulled apart and thus, on the average, away from their equilibrium positions in the interaction potential, which implies a reduction of temperature. But that cannot last. Eventually, irregular holes appear in the fabric of the material and, because the model simulates surface tension, grow (allowing the temperature modestly to increase as the potential energy in nearest-neighbour linkages stretched almost to breaking is converted back into kinetic energy). Finally, as the simulation continues, the outcome is a collection of clusters ('fragments') of varying size and shape so irregular that their definition is hardly child's play.

What matters is whether the outcome is realistic. The simulations (five have been carried through for the purposes of this article) show that the most common fragments are small. In one computer run (nobody says how long it took, or on what kind of machine), the largest cluster contains 90 atoms, but there appear to have been 600 clusters in all, most of them containing just two or three (or even one) atoms.

The logarithm of the number of clusters with more than a specified number of atoms is a decreasing linear function of that number, but there is a sharp change of slope of the curve that argues for a bimodal distribution such that the number of clusters larger than $M$ is of the form $a^{-M / \mu}+b^{-M / k}$, where $a, b, \mu$ and $v$ are constants.

This, according to Holian and Grady, agrees with such experiments as there are. First, there are data gathered by setting off explosive charges in heavy steel cylinders and measuring the sizes of the fragments found and there is also the case of the Universe, where again the logarithm of the number of galaxies whose absolute luminosity exceeds $M$ is a decreasing linear function of $M$ (with no sign of a small galaxy tail in this case).

The authors say that their result conflicts with the old calculation of Mott and Linfoot, who concluded that the distribution would be exponential with diameter. But it is too early to be so sure. There remain gaps in Holian and Grady's argument, not the least of which is that their five simulations all correspond to startingconditions in which there is enough kinetic energy to make the simulated materials liquid. Solids (attainable even in two dimensions with the Lennard-Jones potential) might be different.

That is not to complain. Grady, from Sandia, five years ago used macroscopic energetic considerations to calculate the average mass of fragments on the explosive impulse. It is largely a matter of telling what proportion of the initial kinetic energy will go into the creation of free fragment surface, from which it emerges that average fragment size decreases as the original impulse increases. But the simulations, when the parameters are suitably scaled, yield average sizes smaller by at least an order of magnitude. The explanation, interesting if not surprising, is that the simulated fragments are far from equilibrium (too much surface has been created).

It would be pleasing if the next step were a full three-dimensional simulation. Ordinarily, one might expect the military to be willing to support the most elaborate studies bearing on the way weapons work. Is it possible that they have become smart, and understand that shell casings are really two-dimensional? John Maddox 